\title{
Inhaled corticosteroids might not increase the risk of pneumonia in patients with chronic obstructive pulmonary disease in Japan
}

This article was published in the following Dove Press journal: International Journal of COPD

\section{Ryosuke Hirano \\ Masaki Fujita \\ Takemasa Matsumoto \\ Rintaro On \\ Kentaro Watanabe}

Department of Respiratory Medicine, Fukuoka University Hospital, Nanakuma, Jonanku, Fukuoka, Japan
Correspondence: Masaki Fujita Department of Respiratory Medicine, Fukuoka University Hospital, 7-45-I Nanakuma, Jonanku, Fukuoka 8I4-0I80, Japan

Tel $+8 \mid 92801$ I0I I

Fax +8I 928656220

Email mfujita@fukuoka-u.ac.jp
Background: The use of inhaled corticosteroid (ICS) in patients with chronic obstructive pulmonary disease (COPD) decreases the frequency of COPD exacerbations. Recently, pneumonia was reported as a complication of ICS in patients with COPD. However, there have been few reports concerning the relationship between ICS and pneumonia in Japan. Moreover, there is little information on the types of ICS.

Patients and methods: To clarify these issues, we investigated the occurrence of pneumonia in Japanese patients with COPD. We retrospectively investigated the occurrence of pneumonia in patients with COPD in our hospital from January 2009 to August 2013. Morbidity and mortality, ICS use, age, sex, and COPD classification were investigated. A group of patients with COPD who received ICS and a group of patients with COPD who did not receive ICS were compared each other.

Results: Fifty-one patients developed pneumonia among 639 (7.98\%) patients with COPD. Among 252 ICS-treated patients with COPD, 13 (5.16\%) developed pneumonia, and among 387 ICS-untreated patients with COPD, 38 (9.82\%) developed pneumonia. The mortality rate in ICS-treated patients with COPD was $7.7 \%$, while that in ICS-untreated patients was $10.5 \%$ $(P=0.767)$. Fluticasone/salmeterol use tended to show a higher risk of pneumonia than budesonide/formoterol use. The use of ICS did not increase the risk of pneumonia or mortality due to pneumonia in Japanese patients with COPD.

Conclusion: ICS might not increase the risk of pneumonia in Japanese patients with COPD. In regard to pneumonia, ICS can be safely used in Japanese patients with COPD. Because there are apparent differences in lung diseases among races, appropriate treatment should be investigated in each country.

Keywords: inhaled corticosteroid, adverse event, pneumonia, COPD

\section{Introduction}

Chronic obstructive pulmonary disease (COPD) is characterized by airflow limitation that is not fully reversible. COPD is a major cause of chronic morbidity and mortality worldwide. ${ }^{1}$ Inhaled corticosteroids (ICSs) are important drugs for treating bronchial asthma. Additionally, ICSs are used to treat patients with severe COPD who suffer from repeated exacerbations. Compared with systemic use of corticosteroids, serious adverse events with ICS are rare, but local adverse events, such as oral candidiasis and hoarseness, have been reported..$^{2}$ In recent reports, the forced expiratory volume in 1 second markedly decreased when ICS administration was interrupted. ${ }^{3,4}$ Recently, dual therapy was shown to be inferior to triple therapy for acute exacerbation of COPD. ${ }^{5}$ ICSs are also important drugs for treating COPD. 
ICSs were recently reported to be associated with a high frequency of pneumonia. In the Toward a Revolution in COPD Health (TORCH) study, the ICS group had a significantly higher frequency of pneumonia than did the placebo group in $2007 .{ }^{6}$ Although similar results have been reported since $2007,{ }^{7}$ the effect of ICS on mortality of pneumonia is unclear. Other studies have reported that the risk of pneumonia is not related to ICS use. ${ }^{8,9}$ Moreover, another study showed that use of ICS was not associated with an increased risk of pneumonia in patients with asthma. ${ }^{10}$ Fluticasone/salmeterol (FP/ $\mathrm{SM}$ ) use tends to show a higher risk of pneumonia than does budesonide/formoterol (BUD/FM) use. ${ }^{11}$ Therefore, controversy remains concerning the relationship between ICS and pneumonia. These previous findings were based on studies of Caucasians. Racial differences in the risk of contracting pulmonary diseases, such as drug-induced pneumonia with gefitinib administration, have been well documented. ${ }^{12}$ However, few reports have been published concerning the relationship between ICS and the risk of pneumonia in Japan. Moreover, there is little information on the types of ICS. To clarify the relationship between ICS use and pneumonia in Japanese patients with COPD, we retrospectively analyzed the incidence of pneumonia in patients with COPD at our hospital.

\section{Patients and methods}

\section{Patients}

Patients with COPD were retrospectively investigated at our hospital using medical records. We investigated the medical records from January 2009 to August 2013. The morbidity and mortality rates, ICS use, age, sex, and COPD classification were all evaluated, as was long-term safety. The incidence of pneumonia was monitored for 3 years, as described in the TORCH study. ${ }^{6}$ All patients with pneumonia due to infectious organisms were enrolled. Chest X-rays and chest computed tomography were performed to confirm pneumonia. A group of patients with COPD who received ICS and a group of patients with COPD who did not receive ICS were compared each other.

\section{Risk factors for pneumonia}

The risk factors for pneumonia, besides ICS use, were investigated. We classified ICS-treated patients with COPD into two groups: pneumonia and nonpneumonia groups. These two groups were matched to controls by age, sex, and ICS type for a case-control study. The ratio of patients in the pneumonia and nonpneumonia groups was 1:2. We compared the smoking history, oral steroid administration, oxygen therapy, inoculation with a pneumococcus vaccine, serum albumin levels, and body mass index (BMI) between the two groups.

\section{Ethics of clinical practice}

This study was approved by the local institutional review board of Fukuoka University Hospital (approval number: 15-8-03, approved on September 29, 2015) and was conducted in compliance with the guidelines of good clinical practice and the principles of the Declaration of Helsinki. The patient consent to review their medical records was not required by the local institutional review board of Fukuoka University Hospital since correct number for events are very important and exclusion of any patients limited the scientific validity of the analyses. Moreover, the study poses no clinical risk or harm to the subjects included in the study and any additional risks are limited to those of privacy and confidentiality which are minimal.

\section{Statistical analysis}

Statistical analysis was performed using the chi-squared test or nonparametric analysis (Mann-Whitney $U$ test). A $P$-value $<0.05$ was considered statistically significant. Univariate analyses were performed to select a first set of covariates to be tested for inclusion in a multivariate model, in which all covariates with a $P$-value $<0.2$ by simple logistic regression analysis were included. After completion of univariate analyses, a multiple logistic regression model was selected by the stepwise method. These statistical analyses were performed using the Stata version 9.2 software (StataCorp LLC, College Station, TX, USA).

\section{Results}

A total of 252 patients with COPD had a record of ICS treatment during the study period. The median age was 75 years (range: $47-94$ years), and $84 \%$ were men. Sixty (24\%) patients had been treated for more than 3 years. The types of ICS were as follows: FP/SM was used in 193 patients; BUD/FM in 30; FP in 12; BUD in 2; and others in 15 (Table 1). All of the patients with COPD were smokers.

Fifty-one patients developed pneumonia among the 639 (7.98\%) patients with COPD. Among 252 ICS-treated patients with COPD, 13 developed pneumonia. The incidence of pneumonia among the treated patients was $5.16 \%$. The median age was 80 years (69-94 years), and 92\% were men. Six patients had malignant tumors, two had diabetes, and two had heart failure. Classification of COPD was defined by the Global Initiative for Chronic Obstructive Lung Disease (GOLD) A-D grades, with grade A in two patients, grade B in four, grade $\mathrm{C}$ in one, and grade $\mathrm{D}$ in six (Table 2). 
Table I Demographic and clinical characteristics of patients with COPD

\begin{tabular}{|c|c|c|c|}
\hline Parameters & ICS group $(n=252)$ & Non-ICS ( $n=387)$ & $P$-value \\
\hline Median (range) age (years) & $75(47-94)$ & $73(31-95)$ & 0.074 \\
\hline Gender (male/female) & $211 / 41$ & $337 / 50$ & 0.248 \\
\hline Median (range) number of days of follow-up & $160(12-1,688)$ & $118(1-1,684)$ & 0.631 \\
\hline \multicolumn{4}{|l|}{ Duration of ICS treatment (days) } \\
\hline $0-365$ & 142 & & \\
\hline $366-730$ & 27 & & \\
\hline $73 I-1,095$ & 20 & & \\
\hline Over 1,096 & 60 & & \\
\hline \multicolumn{4}{|l|}{ Type of ICS, n (\%) } \\
\hline FP/SM & $193(76.6)$ & & \\
\hline $\mathrm{BUD} / \mathrm{FM}$ & $30(11.9)$ & & \\
\hline FP & $12(4.8)$ & & \\
\hline BUD & $2(0.8)$ & & \\
\hline Others & $15(6.0)$ & & \\
\hline \multicolumn{4}{|l|}{ Doses of ICS treatment } \\
\hline Low dose & 45 & & \\
\hline Intermediate & 122 & & \\
\hline High & 30 & & \\
\hline Others (changed doses) & 55 & & \\
\hline \multicolumn{4}{|l|}{ Combination of bronchodilators, $n$} \\
\hline LABA & 228 & 188 & $<\mathbf{0 . 0 0 1}$ \\
\hline LAMA & 149 & 170 & $<0.00$ I \\
\hline \multicolumn{4}{|l|}{ Comorbidities, n } \\
\hline Malignant tumor & 72 & 104 & $0.65 I$ \\
\hline Diabetes mellitus & 23 & 51 & 0.130 \\
\hline Ischemic heart disease & 11 & 18 & 1.000 \\
\hline Heart failure & 10 & 27 & 0.122 \\
\hline \multicolumn{4}{|l|}{ GOLD score } \\
\hline A & 32 & 217 & $<0.001$ \\
\hline B & 90 & 88 & 0.233 \\
\hline $\mathrm{C}$ & 14 & 18 & 0.711 \\
\hline $\mathrm{D}$ & 113 & 64 & $<0.00$ I \\
\hline
\end{tabular}

Note: $P$-values in bold $<0.05$ were regarded as statistically significant.

Abbreviations: BUD, budesonide; BUD/FM, budesonide/formoterol; COPD, chronic obstructive pulmonary disease; FP, fluticasone; FP/SM, fluticasone/salmeterol; GOLD, Global Initiative for Chronic Obstructive Lung Disease; ICS, inhaled corticosteroid; LABA, long-acting beta 2 agonist; LAMA, long-acting muscarinic antagonist.

In the 387 ICS-untreated patients with COPD, 38 developed pneumonia. The incidence of pneumonia among the untreated patients was $9.82 \%$. The median age was 78 years (51-91 years), and $89 \%$ were men. Nine patients had malignant tumors, seven patients had diabetes, and three had heart failure. Classification of COPD was defined by GOLD A-D grades, with grade A in 15 patients, grade B in 10 , grade $\mathrm{C}$ in 1 , and grade $\mathrm{D}$ in 12 . In the ICS-untreated group, pneumonia occurred more frequently in grades A and D (Table 2). Especially in grade D, ICS-untreated patients with COPD developed pneumonia more frequently than did ICS-treated patients with COPD $(P=0.019)$.

The risk of pneumonia was higher in ICS-untreated patients than in ICS-treated patients $(P=0.037)$ (Table 3). The rate of pneumonia per observation days was also investigated. The incidence of pneumonia in ICS-treated patients was lower than that in ICS-untreated patients $(P=0.004)$.
This finding indicated that in the pneumonia group, a longer observation did not lead to an increase in the incidence of pneumonia. The relationship of the incidence rate of pneumonia and the observation period was investigated. In the early phase of the follow-up period, pneumonia frequently occurred in ICS-untreated patients. In the late phase, the incidence of pneumonia between ICS-treated and ICSuntreated patients appeared to be similar (Figure 1).

The median period of ICS use was 972 days (29-1,677 days). Ten (77\%) patients were treated with ICS for longer than 1 year, and six patients were treated for longer than 3 years. Eleven patients used FP/SM, one patient used FP, and no patients used BUD/FM or BUD. One patient used ciclesonide. In ICS-untreated patients, long-acting beta 2 agonist or long-acting muscarinic antagonist users were more frequent in the pneumonia group than in the nonpneumonia group. Moreover, in regard to the ICS regimen, pneumonia occurred 
Table 2 Demographic and clinical characteristics of pneumonia in patients with COPD

\begin{tabular}{|c|c|c|c|c|c|c|}
\hline Parameters & \begin{tabular}{|l} 
Pneumonia \\
group in ICS \\
$(n=13)$
\end{tabular} & $\begin{array}{l}\text { Nonpneumonia } \\
\text { in ICS }(n=239)\end{array}$ & $P$-value & $\begin{array}{l}\text { Pneumonia } \\
\text { group in ICS } \\
\text { untreated }(n=38)\end{array}$ & $\begin{array}{l}\text { Nonpneumonia } \\
\text { in ICS untreated } \\
(n=349)\end{array}$ & $\overline{P \text {-value }}$ \\
\hline Median (range) age (years) & $80(69-94)$ & $75(47-94)$ & 0.052 & $78(5|-9|)$ & $72(31-95)$ & 0.009 \\
\hline Gender (male/female) & $12 / 1$ & $199 / 40$ & 0.700 & $34 / 4$ & $303 / 46$ & 0.802 \\
\hline Median (range) number of days of follow-up & $972(29-1,677)$ & $139(12-1,688)$ & 0.010 & $44 \mid(I-I, 680)$ & $100(1-1,684)$ & 0.025 \\
\hline \multicolumn{7}{|l|}{ Duration of ICS treatment (days) } \\
\hline $0-365$ & 3 & 139 & 0.250 & & & \\
\hline $366-730$ & 2 & 25 & 0.637 & & & \\
\hline $731-1,095$ & 2 & 18 & 0.276 & & & \\
\hline Over I,096 & 6 & 54 & 0.087 & & & \\
\hline \multicolumn{7}{|l|}{ Type of ICS, n (\%) } \\
\hline $\mathrm{FP} / \mathrm{SM}$ & II (84.6) & $182(76.2)$ & 0.738 & & & \\
\hline $\mathrm{BUD} / \mathrm{FM}$ & $0(0)$ & $30(12.6)$ & 0.375 & & & \\
\hline FP & I (7.7) & II (4.6) & 0.478 & & & \\
\hline BUD & $0(0)$ & $2(0.8)$ & 1.000 & & & \\
\hline Others & $I(7.7)$ & $14(5.9)$ & 0.559 & & & \\
\hline \multicolumn{7}{|l|}{ Combination of bronchodilators, $n$ (\%) } \\
\hline LABA & II (84.6) & $217(90.8)$ & 1.000 & $28(73.6)$ & $160(45.8)$ & 0.001 \\
\hline LAMA & $8(6 \mid .5)$ & |4| (59.0) & 1.000 & $25(65.7)$ & $145(4 \mid .5)$ & 0.006 \\
\hline \multicolumn{7}{|l|}{ Comorbidities, $n$} \\
\hline Malignant tumor & 6 & 66 & 0.204 & 9 & 95 & 0.705 \\
\hline Diabetes mellitus & 2 & 21 & 0.337 & 7 & 44 & 0.314 \\
\hline Ischemic heart disease & 0 & 11 & 1.000 & 1 & 17 & 1.000 \\
\hline Heart failure & 2 & 8 & 0.088 & 3 & 24 & 0.739 \\
\hline \multicolumn{7}{|l|}{ GOLD score } \\
\hline A & 2 & 30 & 0.673 & 15 & 202 & 0.038 \\
\hline B & 4 & 86 & 0.776 & 10 & 78 & 0.547 \\
\hline C & 1 & 13 & 0.533 & 1 & 17 & 1.000 \\
\hline $\mathrm{D}$ & 6 & 107 & 1.000 & 12 & 52 & 0.019 \\
\hline
\end{tabular}

Note: $P$-values in bold $<0.05$ were regarded as statistically significant.

Abbreviations: BUD, budesonide; BUD/FM, budesonide/formoterol; COPD, chronic obstructive pulmonary disease; FP, fluticasone; FP/SM, fluticasone/salmeterol; GOLD, Global Initiative for Chronic Obstructive Lung Disease; ICS, inhaled corticosteroid; LABA, long-acting beta 2 agonist; LAMA, long-acting muscarinic antagonist.

in $5.70 \%$ of patients in the FP/SM group (11/193 cases) and in $0 \%$ in the BUD/FM group (0/30 cases). These findings indicated that FP/SM use tended to show a higher risk of pneumonia than did BUD/FM use $(P=0.1961)$.

In the ICS-treated group, the median Age-Dehydration, Respiration, Orientation, and Pressure score, which rates the severity of pneumonia determined by the Japan Respiratory

Table 3 Incidence of pneumonia in patients with COPD with or without ICS number of pneumonia cases and observation days in this study

\begin{tabular}{l|l|l|l}
\hline Parameters & $\begin{array}{l}\text { ICS-treated } \\
\text { group }\end{array}$ & $\begin{array}{l}\text { ICS-untreated } \\
\text { group }\end{array}$ & P-value \\
\hline Pneumonia & 13 & 38 & 0.037 \\
\hline Number of cases & 12,324 & 11,249 & 0.004 \\
\hline Total observation (days) & 124 & \\
\hline Without pneumonia & 239 & 349 & \\
\hline Number of cases & 120,679 & 115,461 & \\
\hline Total observation (days) &
\end{tabular}

Abbreviations: COPD, chronic obstructive pulmonary disease; ICS, inhaled corticosteroid.
Society 2005, was $2 .{ }^{13}$ The median number of hospitalized days was 14 (5-33 days). Ten patients recovered, two were transferred to other hospitals, and one died of pneumonia. Both transferred patients finally recovered. In the ICSuntreated group, the median Age-Dehydration, Respiration, Orientation, and Pressure score was 2. The median number of hospitalized days was 11 (6-41 days). Thirtyone patients recovered, three patients were transferred to other hospitals, and four patients died of pneumonia. All transferred patients finally recovered. The mortality rate in ICS-treated patients with COPD was $7.7 \%$, while that in ICS-untreated patients was $10.5 \%(P=0.767)$.

We then compared the incidence of pneumonia by ICS dose. Two patients with low-dose ICS developed pneumonia. Forty-five patients with COPD used a low dose of ICS, and their incidence of pneumonia was $4.44 \%$. The incidence of pneumonia with an intermediate dose of ICS was $3.28 \%$ (4 patients had pneumonia among 122 patients with COPD). The incidence of pneumonia with a high dose of ICS was $10 \%$ (3 patients had pneumonia among 30 patients with COPD). 


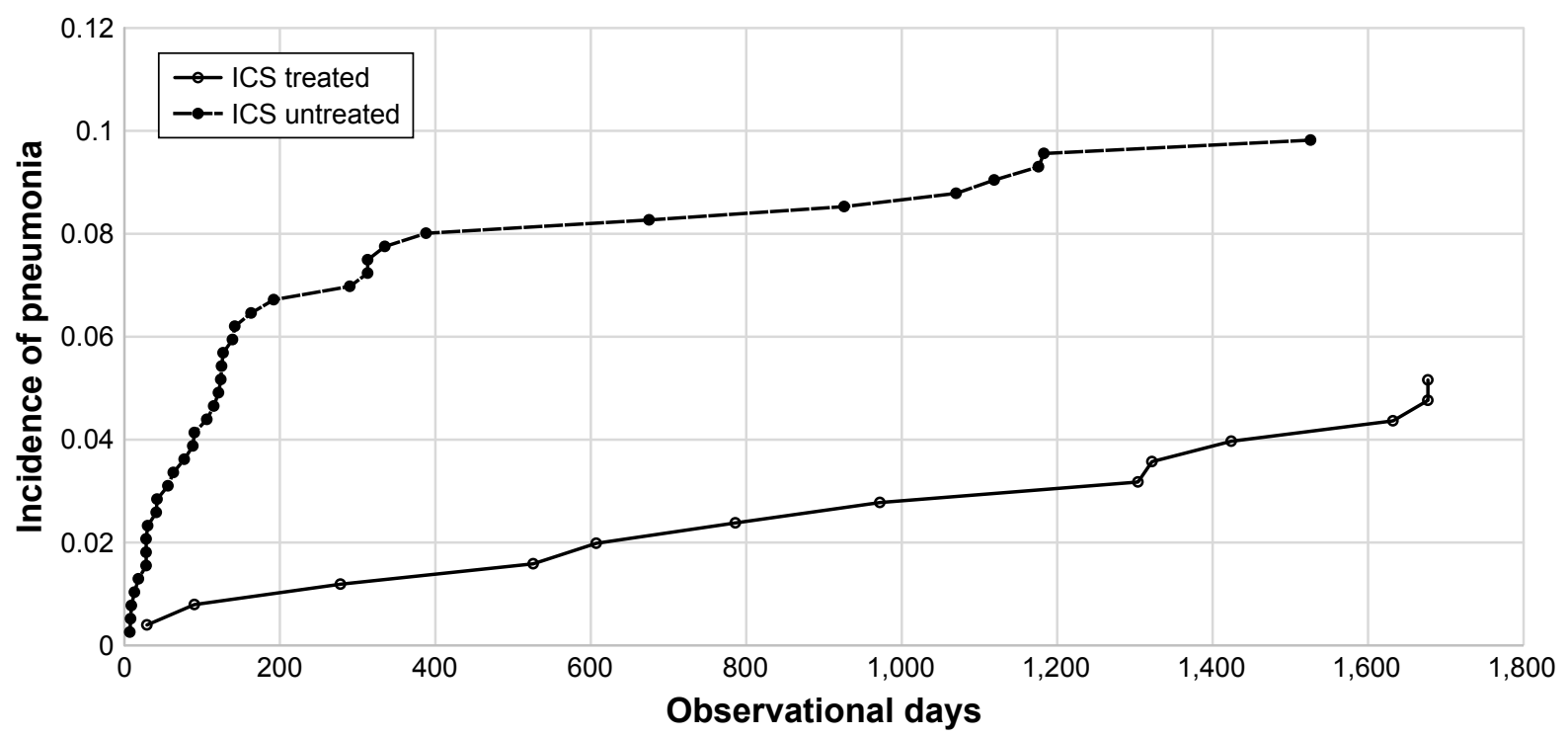

Figure I Incidence rate of pneumonia and the observation period.

Notes: The relationship of the incidence rate of pneumonia and the observation period is shown. The dashed line indicates pneumonia cases in ICS-untreated patients with COPD. The solid line indicates pneumonia cases in ICS-treated patients. In the early phase of the observation period, the rate of pneumonia was high in ICS-untreated patients. In the late phase, the incidence of pneumonia was similar between ICS-treated and ICS-untreated patients.

Abbreviations: COPD, chronic obstructive pulmonary disease; ICS, inhaled corticosteroid.

The incidence rate tended to be high among patients who received a high dose of ICS (10\%), but this was not significant (odds ratio: $0.34,95 \%$ CI: $0.08-1.42, P=0.143$ ). These results indicated a possible relationship between ICS, especially highdose ICS, and pneumonia. The relationship between duration of ICS treatment and pneumonia was not demonstrated.

Among ICS-treated patients, Streptococcus pneumoniae was observed in two, Haemophilus influenzae in one, Moraxella catarrhalis in none, and others in one patient. The bacteria of concern were unknown in nine patients. Among ICS-untreated patients, $S$. pneumoniae was observed in five, $H$. influenzae in five, M. catarrhalis in one, and others in six patients. The bacteria of concern were unknown in 21 patients.

To examine the risk factors of pneumonia besides ICS, we compared the clinical characteristics of 6 patients in the pneumonia group with 12 patients in the nonpneumonia group. The duration of hospitalized days, history of smoking, history of oral corticosteroid, oxygen therapy, history of pneumococcal vaccine, albumin levels, and BMI were compared between the groups. Immunosuppressive medications were not administered. Impairment of lung function was not examined. BMI was significantly lower $(P=0.02)$ in the pneumonia group than in the nonpneumonia group (Table 4 ). However, there was no significance of BMI in multivariate analysis (odds ratio: $0.45,95 \% \mathrm{CI}$ : $0.19-1.02, P=0.055$ ).

\section{Discussion}

In this study, we evaluated the risk of pneumonia in ICStreated Japanese patients with COPD. Although several reports on ICS and pneumonia have been published, to the best of our knowledge, this is the first report to examine the relationship between ICS and pneumonia in patients with COPD in Japan. We found that the incidence of pneumonia

Table 4 Non-ICS risk factors for pneumonia

\begin{tabular}{l|l|l|l|l}
\hline Parameters & $\begin{array}{l}\text { Pneumonia } \\
(\mathbf{n}=\mathbf{6})\end{array}$ & $\begin{array}{l}\text { Nonpneumonia } \\
(\mathbf{n}=\mathbf{1 2})\end{array}$ & $\boldsymbol{P}$-value & $\begin{array}{l}\text { Multivariate analysis, } \\
\boldsymbol{P} \text {-value }\end{array}$ \\
\hline Duration of hospitalization (days) & $1,022.2$ & 945.7 & 0.72 & \\
History of smoking & 6 & 12 & 1 & \\
Using oral corticosteroids & 5 & 10 & 1 & \\
Oxygen therapy & 2 & 3 & 0.71 & \\
Pneumococcal vaccine & 3 & 2 & 0.14 & \\
Albumin (g/dL) & 3.8 & 3.86 & 0.76 & 0.055 \\
BMI & 18.3 & 21.9 & 0.02 & \\
\hline
\end{tabular}

Abbreviations: BMI, body mass index; ICS, inhaled corticosteroid. 
was not high in our patients, especially those receiving BUD/FM, and ICS use did not appear to affect the risk of pneumonia. Moreover, in the GOLD-D score group, ICSuntreated patients with COPD developed pneumonia more frequently than did ICS-treated patients with COPD. In the TORCH study, the ICS group had a significantly higher frequency of pneumonia than did the placebo group. ${ }^{6}$ A plausible explanation for the difference in findings between our study and the TORCH study is that the dose of ICS was smaller in our study than in the TORCH study. We could not demonstrate significance concerning the dose of ICS because we included a small number of patients. However, the dose of ICS could have affected the onset of pneumonia in our study. Additionally, the type of ICS was different between studies. In this study, FP/SM use tended to show a higher risk of pneumonia than did BUD/FM use. Since 30 patients used BUD/FM in our study, a portion of BUD/ FM use might affected lower incidence of pneumonia in our study. We also found that pneumonia occurred in ICSuntreated patients more frequently than ICS-treated patients in the early phase during follow-up (Figure 1). The reason for this finding is unclear. However, ICS could decrease the occurrence of pneumonia in the early phase of induction, or ICS-untreated patients could have comorbidities that are susceptible to pneumonia. In the late phase, the incidence of pneumonia appeared to be similar between ICS-treated and ICS-untreated patients in our study. Another plausible explanation for the difference between studies is a difference in race. Compared with Caucasians, Japanese patients with COPD appear to be older when entering similar clinical trials, and their quality of life scores and prognosis appear better, despite similar pulmonary function impairment. ${ }^{14}$ Racial differences in the risk of contracting pulmonary diseases, such as drug-induced pneumonia with gefitinib administration, have been well documented. ${ }^{12}$ This possibility needs to be investigated in a future study.

Mortality due to pneumonia was not higher in patients who received ICS than in untreated patients. Kew et $\mathrm{al}^{15}$ reported similar mortality results and found that ICS increased the risk of serious adverse pneumonia events but did not significantly affect mortality in treated patients compared with controls. In regard to the risk of pneumonia, ICS is not a risk factor for increasing mortality in Japanese patients with COPD.

Risk factors for pneumonia other than ICS use were also investigated in our study. Our findings suggest that BMI may be a risk factor in patients with COPD, although multivariate analysis did not show a significant difference. Progression of
COPD is associated with loss of body weight. ${ }^{16}$ In our study, although even patients with severe COPD were administered ICS, loss of body weight appeared to be a more influential risk factor than ICS use.

We examined the frequency of pneumonia between patients who received FP/SM and those who received BUD/ FM. FP/SM use tended to increase the risk of pneumonia more than BUD/FM use, which is similar to a previous study. ${ }^{11}$ None of the patients who used BUD/FM developed pneumonia in the present study. Janson et $\mathrm{al}^{11}$ suggested that FP affects intracellular steroid receptors more than BUD, and FP is able to penetrate at the site of action. In this manner, the FP/SM regimen might inhibit the protection mechanism against bacterial infection.

\section{Limitations}

Several limitations that are associated with the present study warrant mention. First, this study was retrospective and is thus less reliable than a prospective study. Second, our study population was relatively small. We conducted our study at a single institution, and only 60 patients had been treated with ICS in the long term ( $>3$ years). The number of cases of pneumonia in our study was also relatively low compared with previous reports. ${ }^{6}$ To examine the risk factors of pneumonia besides ICS, impairment of lung function was not examined. However, recently, ICS was shown to decrease the mortality rate in COPD pneumonia in Japan. ${ }^{17}$ We believe that ICS use is safe in Japan. We are planning to conduct a prospective study in multiple institutions.

\section{Conclusion}

The present study shows that the frequency of pneumonia and mortality is not increased with ICS administration in Japan. ICS might be safely used in Japanese patients with COPD. Appropriate treatment for COPD should be investigated in each country because there are apparent differences in lung diseases among races.

\section{Acknowledgment}

We appreciate the assistance of Edanz Group Japan and Dr Brian Quinn and Dr Ellen Knapp in editing the manuscript and ensuring correct English use. There was no financial support for this study.

\section{Author contributions}

$\mathrm{RH}$ and MF conceived the experiment, carried out all of the experiments, and prepared the manuscript. TM and RO 
cooperated with accumulation of clinical data. KW participated in the study's design, coordination of the study, and final revisions of the manuscript. All authors contributed toward data analysis, drafting and critically revising the paper and agree to be accountable for all aspects of the work. All authors read and approved the final manuscript.

\section{Disclosure}

The authors report no conflicts of interest in this work.

\section{References}

1. Global Initiative for Chronic Obstructive Lung Disease. The Global Strategy for the Diagnosis, Management and Prevention of COPD. Global Initiative for Chronic Obstructive Lung Disease (GOLD); 2017. Available from: http://goldcopd.org. Accessed December 15, 2017.

2. Szafranski W, Cukier A, Ramirez A, et al. Efficacy and safety of budesonide/formoterol in the management of chronic obstructive pulmonary disease. Eur Respir J. 2003;21(1):74-81.

3. Magnussen H, Disse B, Rodriguez-Roisin R, et al. Withdrawal of inhaled glucocorticoids and exacerbations of COPD. NEngl J Med. 2014;371(14): 1285-1294.

4. Kunz LIZ, Postma DS, Klooster K, et al. Relapse in FEV1 decline after steroid withdrawal in COPD. Chest. 2015;148(2):389-396.

5. Lipson DA, Barnhart F, Brealey N, et al. Once-Daily Single-Inhaler Triple versus Dual Therapy in Patients with COPD. N Engl J Med. 2018; 378(18):1671-1680.

6. Crim C, Calverley PM, Anderson JA, et al. Pneumonia risk in COPD patients receiving inhaled corticosteroids alone or in combination: TORCH study results. Eur Respir J. 2009;34(3):641-647.

7. Calverley PM, Stockley RA, Seemungal TA. Investigating New Standards for Prophylaxis in Reduction of Exacerbations (INSPIRE) Investigators. Reported pneumonia in patients with COPD: findings from the INSPIRE study. Chest. 2011;139(3):505-512.
8. Welte T, Miravitlles M, Hernandez P, et al. Efficacy and tolerability of budesonide/formoterol added to tiotropium in patients with chronic obstructive pulmonary disease. Am J Respir Crit Care Med. 2009;180(8): 741-750.

9. Sin DD, Tashkin D, Zhang X, et al. Budesonide and the risk of pneumonia: a meta-analysis of individual patient data. Lancet. 2009; 374(9691):712-719.

10. O'Byrne PM, Pedersen S, Carlsson LG, et al. Risks of pneumonia in patients with asthma taking inhaled corticosteroids. Am J Respir Crit Care Med. 2011;183(5):589-595.

11. Janson C, Larsson K, Lisspers KH, et al. Pneumonia and pneumonia related mortality in patients with COPD treated with fixed combinations of inhaled corticosteroid and long acting $\beta 2$ agonist: observational matched cohort study (PATHOS). BMJ. 2013;346:f3306.

12. Inoue A, Saijo Y, Maemondo M, et al. Severe acute interstitial pneumonia and gefitinib. Lancet. 2003;361(9352):137-139.

13. Kohno S, Seki M, Watanabe A; CAP Study Group. Evaluation of an assessment system for the JRS 2005: A-DROP for the management of CAP in adults. Intern Med. 2011;50(11):1183-1191.

14. Ichinose $\mathrm{M}$, Taniguchi $\mathrm{H}$, Takizawa $\mathrm{A}$, et al. The efficacy and safety of combined tiotropium and olodaterol via the Respimat( $(\mathbb{R})$ inhaler in patients with COPD: results from the Japanese sub-population of the Tonado(®) studies. Int J Chron Obstruct Pulmon Dis. 2016;11: 2017-2027.

15. Kew KM, Seniukovich A. Inhaled steroids and risk of pneumonia for chronic obstructive pulmonary disease. Cochrane Database Syst Rev. 2014;3:CD010115.

16. Vestbo J, Prescott E, Almdal T, et al. Body mass, fat-free body mass, and prognosis in patients with chronic obstructive pulmonary disease from a random population sample: findings from the Copenhagen City Heart Study. Am J Respir Crit Care Med. 2006;173(1):79-83.

17. Yamauchi Y, Yasunaga H, Hasegawa W, et al. Effect of outpatient therapy with inhaled corticosteroids on decreasing in-hospital mortality from pneumonia in patients with COPD. Int $J$ Chron Obstruct Pulmon Dis. 2016;11:1403-1411.
International Journal of COPD

\section{Publish your work in this journal}

The International Journal of COPD is an international, peer-reviewed journal of therapeutics and pharmacology focusing on concise rapid reporting of clinical studies and reviews in COPD. Special focus is given to the pathophysiological processes underlying the disease, intervention programs, patient focused education, and self management protocols.

\section{Dovepress}

This journal is indexed on PubMed Central, MedLine and CAS. The manuscript management system is completely online and includes a very quick and fair peer-review system, which is all easy to use. Visit $\mathrm{http} / / / \mathrm{www}$.dovepress.com/testimonials.php to read real quotes from published authors. 